\title{
Magneto-seismology of solar atmospheric loops by means of longitudinal oscillations
}

\author{
M. Luna-Cardozo ${ }^{1,3}$, G. Verth $^{2}$ and R. Erdélyi ${ }^{3}$ \\ ${ }^{1}$ Instituto de Astronomía y Física del Espacio (IAFE), CONICET-UBA, \\ CC. 67, Suc. 28, 1428 Buenos Aires, Argentina. email: mluna@iafe.uba.ar \\ ${ }^{2}$ School of Computing, Engineering and Information Sciences, Northumbria \\ University, Newcastle Upon Tyne, NE1 8ST, UK. email: gary.verth@northumbria.ac.uk \\ ${ }^{3}$ Solar Physics and Space Plasma Research Centre $\left(\mathrm{SP}^{2} \mathrm{RC}\right)$, University of Sheffield, \\ Hicks Building, Hounsfield Road, Sheffield S3 7RH, UK. email: robertus@sheffield.ac.uk
}

\begin{abstract}
There is increasingly strong observational evidence that slow magnetoacoustic modes arise in the solar atmosphere. Solar magneto-seismology is a novel tool to derive otherwise directly un-measurable properties of the solar atmosphere when magnetohydrodynamic (MHD) wave theory is compared to wave observations. Here, MHD wave theory is further developed illustrating how information about the magnetic and density structure along coronal loops can be determined by measuring the frequencies of the slow MHD oscillations. The application to observations of slow magnetoacoustic waves in coronal loops is discussed.
\end{abstract}

Keywords. (magnetohydrodynamics:) MHD, Sun: corona, Sun: fundamental parameters, Sun: magnetic fields, Sun: oscillations, Waves

\section{Introduction}

Damped slow MHD oscillations have been observed in the solar atmosphere using high-resolution EUV imager on board space-borne telescopes (see review by Wang 2011). Such oscillations are important because of their potential for the diagnostics of magnetic structures by implementation of the method of magneto-seismology, through matching the MHD wave theory and wave observations in the solar atmosphere to obtain several physical parameters (e.g., magnetic field strength and density scale height).

The theory of MHD wave propagation in solar magnetic structures initially began modelling the magnetic structures as homogeneous cylindrical magnetic flux tubes enclosed within a magnetic environment (Roberts et al. 1984). Later on, more advanced equilibrium models to study slow MHD oscillations have also been proposed with e.g., dissipative effects and gravity (Mendoza-Briceño et al. 2004, Sigalotti et al. 2007), and non-isothermal profiles (Erdélyi et al. 2008), while the effect of density and magnetic stratification had been revisited on transversal coronal loop oscillations by Dymova \& Ruderman (2006) and Verth \& Erdélyi (2008), respectively.

Here, the governing equation of the longitudinal mode is solved numerically for density stratified loops with uniform magnetic field, as well as for expanding magnetic flux tubes with uniform density. The effect of these stratifications on the frequency ratio of the first overtone to the fundamental mode is studied.

\section{Governing equation}

The ideal MHD equations are linearized by considering small magnetic and velocity perturbations about a plasma in static equilibrium $\left[\vec{b}=\left(b_{r}, 0, b_{z}\right)\right.$ and $\vec{v}=\left(v_{r}, 0, v_{z}\right)$, 
for $r$ and $z$ the radial and longitudinal coordinates, respectively]. In the derivation, a uniform kinetic plasma pressure is assumed, and the thin flux tube approximation is considered. The second-order ordinary differential equation governing the longitudinal velocity amplitude is (see Luna-Cardozo et al. 2012 for a detailed derivation)

$$
\frac{d^{2} v_{z}}{d z^{2}}+\left(\frac{c_{\mathrm{s}}^{2}-c_{\mathrm{A}}^{2}}{c_{\mathrm{f}}^{2}}\right) \frac{1}{B_{z}} \frac{\partial B_{z}}{\partial z} \frac{d v_{z}}{d z}+\left[\frac{\omega^{2}}{c_{\mathrm{T}}^{2}}-\frac{1}{B_{z}} \frac{\partial^{2} B_{z}}{\partial z^{2}}-\left(\frac{c_{\mathrm{s}}^{2}-c_{\mathrm{A}}^{2}}{c_{\mathrm{f}}^{2}}\right) \frac{1}{B_{z}^{2}}\left(\frac{\partial B_{z}}{\partial z}\right)^{2}\right] v_{z}=0
$$

where $c_{\mathrm{A}}^{2}=\left(B_{z}^{2} / \mu \rho_{0}\right), c_{\mathrm{s}}^{2}=\left(\gamma p_{0} / \rho_{0}\right), c_{\mathrm{f}}^{2}=c_{\mathrm{s}}^{2}+c_{\mathrm{A}}^{2}$ and $c_{\mathrm{T}}^{2}=\left(c_{\mathrm{s}}^{-2}+c_{\mathrm{A}}^{-2}\right)^{-1}$ are the square of the Alfvén, sound, fast phase and tube speeds, respectively. In this equation, $\omega$ is the angular frequency of the oscillations.

Equation (2.1) is numerically solved using the shooting method based on the RungeKutta technique, for density stratified loops with uniform magnetic field, as well as for expanding loops with uniform density. Solar waveguides are modelled as axisymmetric cylindrical magnetic tubes with tube ends frozen in a dense photospheric plasma at $z= \pm L$. On average, plasma density and magnetic field strength are expected to decrease with height above the photosphere (Lin et al. 2004).

\section{Effect of density stratification}

The solar coronal loop is modelled by a straight axisymmetric magnetic flux tube with tube length of $2 L$ and radius of $r_{0}$. The uniform magnetic field is directed along the tube axis, i.e., $\vec{B}=B_{z} \hat{z}$. In semi-circular coronal loops where the plasma is close to hydrostatic equilibrium, a reasonable assumption for the density profile is

$$
\rho_{0}(z)=\rho_{\mathrm{f}} \exp \left[-\frac{2 L}{\pi H} \cos \left(\frac{\pi z}{2 L}\right)\right]
$$

where $H$ is the density scale height and $\rho_{\mathrm{f}}$ the density at the footpoint. To study a standing wave the boundary condition $v_{z}( \pm L)=0$ is applied. We solve equation (2.1) using the density profile (3.1). The frequency ratio of the first overtone to the fundamental mode is shown in Figure 1(a) as a function of $L / H$ by the solid line, and it is clearly lower than the canonical value of two. A similar result was obtained for the transversal mode by Dymova \& Ruderman (2006) and Verth (2007).

For vertical chromospheric flux tubes the density profile is given by

$$
\rho_{0}(z)=\rho_{\mathrm{f}} \exp \left[-\frac{(z+L)}{H}\right] .
$$

Longitudinal oscillations in chromospheric flux tubes are studied solving the eigenvalue problem in half of the magnetic bottle, i.e., designating $v_{z}(-L)=v_{z}(0)=0$ as the boundary conditions. The ratio of frequencies against $L / H$ for the density profile (3.2) is shown by the dashed line in Figure 1(a). Now, the frequency ratio is slightly greater than two, indicating that this parameter depends on the functional form chosen of the equilibrium density. This suggests that caution must be used when interpreting the frequency ratio of chromospheric standing modes.

\section{Effect of a non-uniform magnetic field}

An expanding flux tube with rotational symmetry about the $z$-axis in cylindrical coordinates $(r, \theta, z)$ is used to model a magnetic field equilibrium decreasing in strength with 

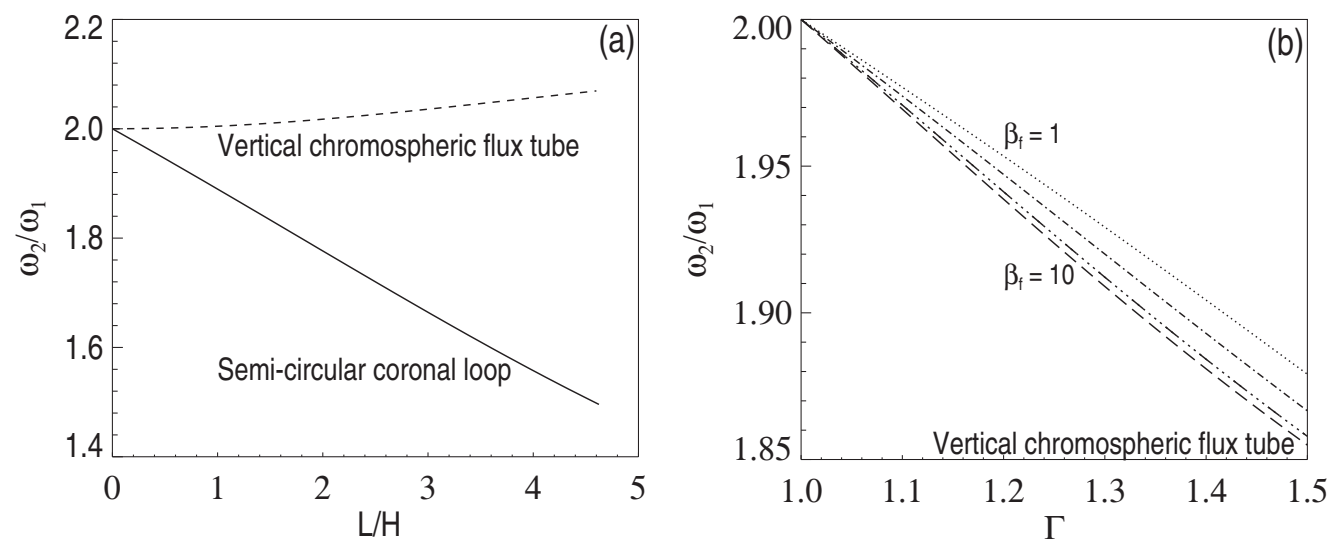

Figure 1. (a) Frequency ratio of the first overtone and fundamental mode against $L / H$ for density stratified coronal (solid line) and chromospheric (dashed line) loops. (b) Frequency ratio against the expansion parameter $\Gamma$ for different values of $\beta_{\mathrm{f}}$ in vertical chromospheric flux tubes. In (b) dotted, dot-dashed, dot-dot-dot-dashed and long-dashed lines correspond to $\beta_{\mathrm{f}}=1,2,5$ and 10 , respectively.

height above the photosphere. The magnetic field component $B_{z}$ at the tube boundary can be described explicitly as function of $z$ (see Verth \& Erdélyi 2008)

$$
B_{z}(z) \approx B_{z, \mathrm{f}}\left\{1+\frac{\left(1-\Gamma^{2}\right)}{\Gamma^{2}} \frac{[\cosh (z / L)-\cosh (1)]}{1-\cosh (1)}\right\},
$$

where $\Gamma=r_{\mathrm{a}} / r_{\mathrm{f}}$ is the expansion factor, and $r_{\mathrm{a}}\left(r_{\mathrm{f}}\right)$ is the apex (footpoint) radius. The loop expansion has been estimated for various loops, giving mean values of $\Gamma \approx 1.16$ and 1.30 for EUV and soft X-ray loops (Watko \& Klimchuk 2000, Klimchuk 2000).

We can compute the numerical solution of equation (2.1) for slow longitudinal oscillations in coronal and chromospheric loops setting the same boundary conditions as in the previous section, and using equation (4.1) for $B_{z}(z)$. Figure 1(b) shows the frequency ratio as function of the expansion parameter $\Gamma$ for different values of the footpoint beta plasma $\beta_{\mathrm{f}}$ for chromospheric flux tubes. It is found that when the magnetic expansion factor increases the frequency ratio decreases, and this effect is more significant for chromospheric flux tubes with higher $\beta_{\mathrm{f}}$.

Figure 2 shows the frequency ratio as function of the expansion factor for coronal loops with uniform density in (a) and for typical density stratification (i.e., $L / H=2$ ) in (b). It can be seen how these two effects, density stratification and magnetic expansion, contribute to decrease the value of $\omega_{2} / \omega_{1}$. Additionally, the effect of the expansion is stronger in the corona than in the chromosphere.

\section{Summary and conclusions}

Studying the solutions of the velocity governing equation of the slow standing mode, it is found that density stratification and magnetic expansion cause the same qualitative effect on the frequency ratio in coronal loops, giving values of $\omega_{2} / \omega_{1}<2$. For chromospheric flux tubes density stratification and magnetic expansion cause opposite effects on the frequency ratio; however, caution must be taken when studying chromospheric flux tubes since the ratio $\omega_{2} / \omega_{1}$ depends on the functional form chosen for the density (see Luna-Cardozo et al. 2012 for an analytical and numerical detailed study about important issues). 

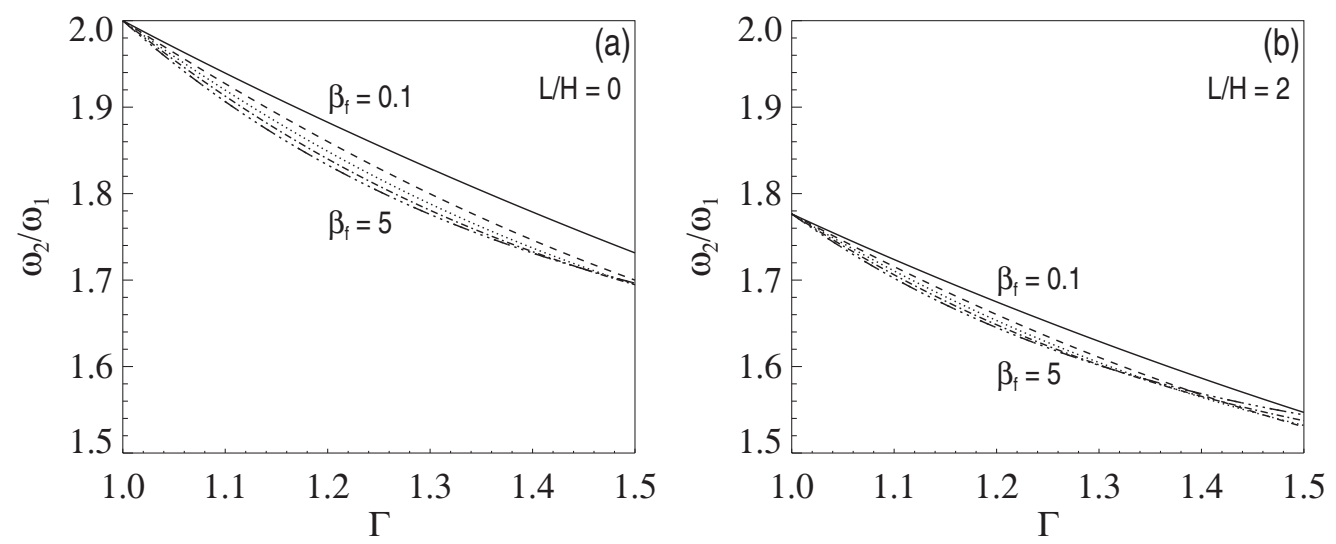

Figure 2. Frequency ratio of coronal loop oscillations against the expansion parameter $\Gamma$ for different values of $\beta_{\mathrm{f}}$. Solid, dashed, dotted, dot-dashed and dot-dot-dot-dashed lines correspond to $\beta_{\mathrm{f}}=0.1,0.5,1,2$ and 5 , respectively. Coronal loops with uniform density $(L / H=0)$ are presented in (a) and with typical density stratification $(L / H=2)$ in (b).

These results are consistent with the values of period ratio of $P_{1} / P_{2}=1.54$ and 1.84 reported by Srivastava \& Dwivedi (2010) while observing slow acoustic oscillations using Hinode, in contrast to the theoretical value of $P_{1} / P_{2}=2$ for a uniform cylindrical flux tube model.

Our results are important for magneto-seismology, where the density scale height of the solar atmosphere can be calculated by using the observed value of the frequency ratio $\omega_{2} / \omega_{1}$ of longitudinal loop oscillations, to complement both emission measure and magnetic field extrapolation studies. This could provide us with a more complete understanding of the plasma fine structure in the solar atmosphere. These results can be applied in any stage of the solar cycle, including the solar minimum.

\section{Acknowledgements}

ML-C thanks the IAU for the travel grant and is grateful for the financial support from PICT 2007-1790 grant (ANPCyT). RE acknowledges M. Kéray for patient encouragement and is also grateful to NSF, Hungary (OTKA, Ref. No. K83133) for financial support received.

\section{References}

Dymova, M. V. \& Ruderman M. S. 2006, A\&A, 457, 1059

Erdélyi, R., Luna-Cardozo, M., \& Mendoza-Briceño, C. A. 2008, Sol. Phys., 252, 305

Klimchuk, J. A. 2000, Sol. Phys., 193, 53

Lin, H., Khun, J. R., \& Coulter, R. 2004, ApJ, 613, L177

Luna-Cardozo, M., Verth, G., \& Erdélyi, R. 2012, ApJ, 748, 110

Mendoza-Briceño, C. A., Erdélyi, R., \& Sigalotti, L. Di G. 2004, ApJ, 605, 493

Roberts, B., Edwin, P. M., \& Benz, A. O. 1984, ApJ, 279, 857

Sigalotti, L. Di G., Mendoza-Briceño, C. A., \& Luna-Cardozo, M. 2007, Sol. Phys., 246, 187

Srivastava, A. K. \& Dwivedi, B. N. 2010, New Astron., 15, 8

Verth, G. 2007, Astron. Nachr., 328, 764

Verth, G. \& Erdélyi, R. 2008, A\& A, 486, 1015

Wang, T. J. 2011, Space Sci. Rev., 158, 397

Watko, J. A. \& Klimchuk J. A. 2000, Sol. Phys., 193, 77 